\title{
Vascular segmentation in hepatic CT images using adaptive threshold fuzzy connectedness method
}

\author{
Xiaoxi Guo ${ }^{1,2 \dagger}$, Shaohui Huang ${ }^{1 * \dagger}$, Xiaozhu Fu ${ }^{1 \dagger}$, Boliang Wang ${ }^{1 \dagger}$ and Xiaoyang Huang ${ }^{1 \dagger}$
}

*Correspondence: hsh@xmu. edu.cn

${ }^{+}$Xiaoxi Guo, Shaohui

Huang, Xiaozhu Fu, Boliang

Wang and Xiaoyang Huang contributed equally to this work

${ }^{1}$ Computer Science

Department, Xiamen

University, Xiamen, China

Full list of author information

is available at the end of the article

\begin{abstract}
Background: Fuzzy connectedness method has shown its effectiveness for fuzzy object extraction in recent years. However, two problems may occur when applying it to hepatic vessel segmentation task. One is the excessive computational cost, and the other is the difficulty of choosing a proper threshold value for final segmentation.

Methods: In this paper, an accelerated strategy based on a lookup table was presented first which can reduce the connectivity scene calculation time and achieve a speed-up factor of above 2 . When the computing of the fuzzy connectedness relations is finished, a threshold is needed to generate the final result. Currently the threshold is preset by users. Since different thresholds may produce different outcomes, how to determine a proper threshold is crucial. According to our analysis of the hepatic vessel structure, a watershed-like method was used to find the optimal threshold. Meanwhile, by using Ostu algorithm to calculate the parameters for affinity relations and assigning the seed with the mean value, it is able to reduce the influence on the segmentation result caused by the location of the seed and enhance the robustness of fuzzy connectedness method.
\end{abstract}

Results: Experiments based on four different datasets demonstrate the efficiency of the lookup table strategy. These experiments also show that an adaptive threshold found by watershed-like method can always generate correct segmentation results of hepatic vessels. Comparing to a refined region-growing algorithm that has been widely used for hepatic vessel segmentation, fuzzy connectedness method has advantages in detecting vascular edge and generating more than one vessel system through the weak connectivity of the vessel ends.

Conclusions: An improved algorithm based on fuzzy connectedness method is proposed. This algorithm has improved the performance of fuzzy connectedness method in hepatic vessel segmentation.

Keywords: Hepatic vessel segmentation, Fuzzy connectedness method, Fuzzy affinity, Adaptive threshold

\section{Background}

In the diagnosis and study of vascular-related diseases, the structure and morphology of the hepatic vessels provide vital information. Especially for surgical planning and outcome assessment of liver operations, such as living-related liver transplants and oncologic 
resections, it is crucial to present a patient-individual 3D structure of the liver along with its vasculature and lesions. Therefore, to achieve an accurate and robust extraction of the intrahepatic vessels is an essential step and plays an important role in medical image analysis.

Due to the low contrast between the blood vessels and the surrounding liver parenchyma, the complex morphology as well as the pathologies of the hepatic vessel system, vascular segmentation is a challenging task and has gained increasing attention and interest. Several specific methods have been proposed to segment vessels in hepatic CT images in recent years [1-5]. Most methods are histogram based, region growing based, level-set based, and geometrical model based.

A theory of fuzzy objects for $n$-dimensional digital spaces based on a notion of fuzzy connectedness of image elements and algorithms for extracting a specified fuzzy object were presented in [6]. Since then, this fuzzy connectedness framework and its extensions have been extensively utilized in many medical applications. Based on an improved fuzzy connectedness algorithm, Harati et al. developed a fully automatic and accurate method for tumor region detection and segmentation in brain MR images [7]. Lloréns et al. used fuzzy-connectedness and morphological processing to segment jaw tissues in dental 3D CT images [8]. Ciesielski et al. jointed graph cut and relative fuzzy connectedness to segment bones from CT images [9]. Badura et al. combined fuzzy connectedness and the evolutionary computation to segment 3D lung nodule [10]. When using fuzzy connectedness method in liver vessel segmentation task, two problems occurred. One is the excessive computational cost, and the other is the difficulty of choosing a proper threshold value for final segmentation. The work in Ref. [11] focused on minimize the computation of fuzzy connectedness relations by pre-determined thresholds for fuzzy objects. However, this pre-determined threshold method is not applicable in hepatic vessel segmentation. In this paper, we present an accelerated strategy to reduce the connectivity scene calculation time and an adaptive threshold based on watershed-like method to classify the connectivity scene in "Methods". The materials utilized and the experiment results are detailed in "Results". We state our conclusion in "Conclusions".

\section{Methods}

\section{Fuzzy adjacency and affinity}

We refer to a 3D hepatic CT image as a scene $C$. The voxels in CT image have a fuzzy adjacency relation, denoted by $\alpha$. The adjacency relation assigns every pair $(c, d)$ of voxels a value between zero and one, denoted by $\mu_{\alpha}(c, d)$. The closer $c$ and $d$ are spatially to each other, the greater is this value. In this paper, we used the 6-adjacency relation for $\alpha$. That is,

$$
\mu_{\alpha}(c, d)= \begin{cases}1, & \text { if } \sqrt{\sum_{i}\left(c_{i}-d_{i}\right)^{2}} \leq 1 \\ 0, & \text { otherwise. }\end{cases}
$$

Another local fuzzy relation called fuzzy affinity is denoted by $k$. For any voxels $c$ and $d$, the strength of this relation, denoted by $\mu_{k}(c, d)$, lies between zero and one, and indicates how the voxels "hanging together" locally in the scene. The general form of $\mu_{k}(c, d)$ is

$$
\mu_{k}(c, d)=\mu_{\alpha}(c, d)\left[\omega_{1} h_{1}(f(c), f(d))+\omega_{2} h_{2}(f(c), f(d))\right],
$$


where $f(x)$ is the Hounsfield unit (HU) scale of voxel $x ; h_{1}$ and $h_{2}$ are scalar-valued functions with range[0,1]; $\omega_{1}$ and $\omega_{2}$ are free parameters satisfying:

$$
\omega_{1}+\omega_{2}=1
$$

Through tests and comparisons, we chose $h_{1}(f(c), f(d))=e^{-\frac{1}{2}\left[\left(\frac{f(c)+f(d)}{2}-m\right) / s\right]^{2}}, \omega_{1}=1$, and $\omega_{2}=0$ in our study. Hence, the affinity relation in this paper is

$$
\mu_{k}(c, d)=\mu_{\alpha}(c, d) \cdot e^{-\frac{1}{2}\left[\left(\frac{f(c)+f(d)}{2}-m\right) / s\right]^{2}},
$$

where $m$ and $s$ represent the mean and standard deviation of voxel values in the object of interest.

\section{Fuzzy connectedness}

The global fuzzy relation on voxels is called fuzzy connectedness, denoted $K$. The strength of this relation $\mu_{K}(c, d)$ lies between zero and one. To assign a connectedness value to any pair of voxels $(c, d)$ needs taking all possible paths between $c$ and $d$ into consideration. Each path is a sequence of links formed between successive voxels, starting from $c$ and ending in $d$. The strength of a path is the weakest pairwise voxel affinity in it. And the fuzzy connectedness strength between $c$ and $d$ is the strength of the strongest of all paths. Assuming that there are $n$ paths $\left(p_{c l}^{1} p_{c l}^{2} \ldots, p_{c d}^{n}\right.$ from $c$ to $d$. A path $p_{c d}^{i}$ consists of voxels $<c, a^{(1)}, a^{(2)}, \ldots, a^{(m)}, d>$.

The strength of a path $\mu_{K}\left(p_{c t}^{i}\right.$ and connectedness of $(c, d)$ are described as follows:

$$
\begin{aligned}
& \mu_{K}\left(p_{c d}^{i}\right)=\min \left[\mu_{k}\left(c, a^{(1)}\right), \mu_{k}\left(a^{(1)}, a^{(2)}\right), \ldots, \mu_{k}\left(a^{(m)}, d\right)\right] \\
& \mu_{K}(c, d)=\max \left[\mu_{K}\left(p_{c d}^{1}\right), \mu_{K}\left(p_{c d}^{2}\right), \ldots, \mu_{K}\left(p_{c d}^{n}\right)\right] .
\end{aligned}
$$

\section{The parameters for affinity relation}

The fuzzy segmentation result is highly correlated to the mean value $m$ and standard deviation value $s$ in (4). Therefore, it is the most important step to obtain these parameters before starting a connectivity scene calculation. Most current algorithms used a relatively small space around the seed to estimate $m$ and $s$ for the intended object, hence the $m$ and $s$ are sensitive to the location of the seed. Due to the low contrast between the blood vessels and liver parenchyma, and the small vessel diameter, the manual selection of the seed may have great impact on whether the segmentation result is accurate. For example, the results from a seed in the center of the vessel and the one on the edge are distinguishing.

To reduce the influence of the seed position and enhance the robustness of fuzzy connectedness method, we use a cube $(20 \times 20 \times 20$ voxels $)$ to enclose the seed in the center of it. In this cube, it would contain vessel as well as other tissues such as liver parenchyma. The Ostu algorithm [12] is utilized to achieve a threshold to classify all voxels in the cube into two groups (the vessels and others). Then $m$ and $s$ of the vessel group are calculated and we replace the value of the seed with $m$. 


\section{A lookup table}

During the connectivity scene calculation, the computational cost is determined mainly by evaluation of affinity relations. As the HU values of vessels are discrete and always lie in 100-230, same voxel pairs would be calculated repeatedly for many times. To speed up this procedure, a lookup table is introduced. At the beginning of fuzzy connectedness algorithm, a table of $256 \times 256$ would be filled at first. This table contains the $\mu_{k}(c, d)$ of the voxel pair $(c, d)$ with HU value $(f(c), f(d))$ ranging from $(0,0)$ to $(255,255)$, which covers all the possible combination of vessel voxel pairs. Every time when the value of $\mu_{k}(c, d)$ is needed, it can just be retrieved from the lookup table and does not need to be calculated again.

\section{The algorithm based on fuzzy connectedness method for hepatic vessel segmentation}

The fuzzy connectedness algorithm for vascular segmentation needs the specification of a seed to build the affinity relations between other voxels and itself, and then to calculate a connectivity scene. Using a threshold, it can produce a segmentation result.

The algorithm based on fuzzy connectedness method for hepatic vessel segmentation is presented as follows:

Input: a scene $C$, a seed $S$

Output: vessel segmentation result $V$

Auxiliary Data Structures: A 3D array representing the connectivity scene CS and a queue $Q$ contains voxels to be processed.

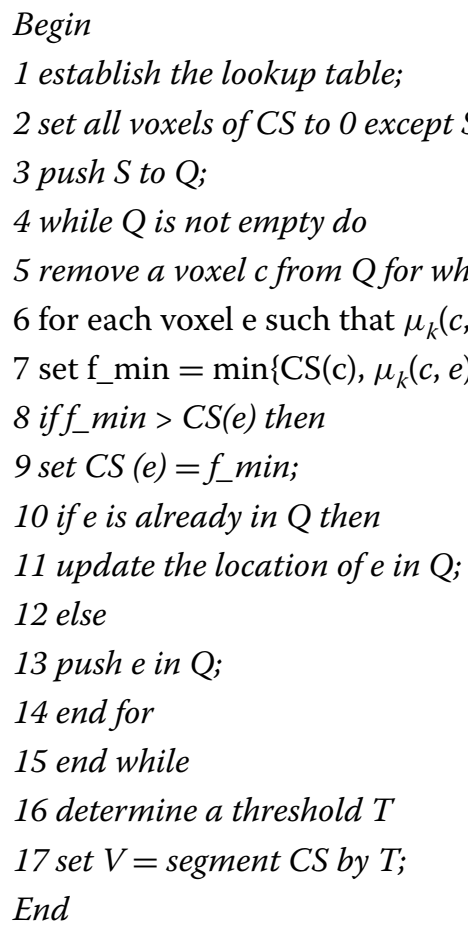

\section{Adaptive threshold}

In the step 16 of the algorithm, a threshold $T$ is used to filter the connectivity scene $C S$ and generate the final result. Currently this threshold can only be preset by users. To choose 
the threshold automatically, the histogram of $C S, H(x)$, should be calculated first. Given a threshold $h$, total volume of segmented object of interest can be expressed as follows:

$$
\operatorname{volume}(h)=\text { voxelsize } * \int_{h}^{1.0} H(x) d x
$$

The CT values of hepatic vessel were very similar and this makes them "hanging together" easily and results in obvious peaks in the histogram. The proper threshold can be calculated from these peaks. The idea of adaptive threshold searching method is as follows: the affinity value of scene represents the similarity of voxels; a high affinity value means a voxel is more similar to the seed. Because the seed lies in the vessel, voxels lying in the same vessel may achieve similar high affinity value. This feature would present as an obvious peak in the histogram. The terminals of blood vessels would reflect a weak connectivity of different vessels in liver CT image, so the histogram may contain many valleys before next peak appears. Another peak indicates that the connection has spread into another vessel. This procedure would be stopped when it reaches the possible maximum volume of hepatic vessels.

Based on the manual measurements of hepatic portal vein from $10 \mathrm{CT}$ datasets shown in Table 1, the average volume of hepatic portal vein is $11.578 \pm 1.684 \mathrm{ml}$.

According to the volume of hepatic portal vein and taking hepatic vein, artery and portal vein into consideration, $50 \mathrm{ml}$ is chosen as the maximum volume of hepatic vessels. To find the proper threshold, a watershed-like method is used in our study. Based on above description, the adaptive threshold finding method is presented as below.

Input: a connectivity scene CS

Output: a suggested threshold T

Auxiliary Data Structures: An array H representing the histogram of CS

Begin

1 calculated $H$ from $C S$;

2 found an endpoint $\mathrm{EP}$ from $\mathrm{H}$, where $\mathrm{EP}=\max (\mathrm{h})$ and satisfiesvolume $(h) \geq 50 \mathrm{ml}$

3 if $E P$ is a peak then

4 let $T=E P$

5 else

6 search from EP to 1.0 until find a peak P;

Table 1 Statistics of Hepatic portal vein volume

\begin{tabular}{llll}
\hline Dataset & Voxel size $(\mathbf{m m})$ & $\begin{array}{l}\text { Num of hepatic } \\
\text { portal vein voxels }\end{array}$ & $\begin{array}{l}\text { Volume of hepatic } \\
\text { portal vein }(\mathbf{m l})\end{array}$ \\
\hline 1 & $0.671 \times 0.671 \times 1$ & 24,904 & 11.213 \\
2 & $0.702 \times 0.702 \times 1$ & 23,297 & 11.481 \\
3 & $0.677 \times 0.677 \times 1$ & 22,370 & 10.253 \\
4 & $0.637 \times 0.637 \times 1$ & 30,421 & 12.344 \\
5 & $0.61 \times 0.61 \times 1$ & 31,539 & 11.736 \\
6 & $0.782 \times 0.782 \times 1$ & 16,179 & 9.894 \\
7 & $0.663 \times 0.663 \times 1$ & 24,073 & 10.582 \\
8 & $0.702 \times 0.702 \times 1$ & 27,623 & 13.613 \\
9 & $0.782 \times 0.782 \times 1$ & 22,304 & 13.640 \\
10 & $0.668 \times 0.668 \times 1$ & 24,714 & 11.028 \\
\hline
\end{tabular}


7 let $T=P$;

8 endif

End

\section{Results}

Our algorithm was implemented in $\mathrm{C}++$, and tests were performed with 4 different sizes of datasets acquired by different $\mathrm{CT}$ machines. Testing computer runs Windows XP, which has an Intel Core i5 CPU and 4 GB of main memory.

Table 2 lists the image datasets information used in our experiments and the performance of the implementation. It shows that the lookup table can speed up the computation of fuzzy connectedness relations by a factor of above 2 .

A refined region-growing algorithm (RGG) presented in [1] has been widely used for intrahepatic vessel segmentation. Starting with a seed of the vessel with intensity $\theta_{\text {beg }}$, RRG iteratively accumulates the 26 adjacent voxels with an intensity equal to or greater than $\theta_{b e g}$ and keeps them in a list $L\left(\theta_{b e g}\right)$, and then uses $L\left(\theta_{b e g}\right)$ as new seeds to collect all adjacent voxels with intensity greater than or equal to $\theta_{\text {beg }}-1$ in a list $L\left(\theta_{\text {beg }}-1\right)$ until a given intensity $\theta_{\text {end }}$ is reached that creates voxels $L\left(\theta_{\text {end }}\right)$ outside the vessel. From $\theta_{\text {end }}$ to $\theta_{\text {beg }}$, the number of voxels $N(\theta)$ is decreasing and changes considerably at $\theta_{\text {opt }}$ for most voxels belong to liver tissue are collected for thresholds below $\theta_{\text {opt }}$. RRG uses the threshold $\theta_{\text {opt }}$ for vessel segmentation. RRG's segmentation result is high related to the starting seed's location, and its running time mostly depends on $\theta_{\text {beg }}$ and $\theta_{\text {end }}$.

We applied RRG to these four datasets using the same seeds. Table 3 shows the quantitative comparisons between RRG and our method. Let $A$ be the segmentation result using our method and $B$ be the segmentation result using RRG, a different set $C$ is the

Table 2 Dataset information and segmentation performance using fuzzy connectedness method without/with a lookup table

\begin{tabular}{|c|c|c|c|c|}
\hline Dataset & 1 & II & III & IV \\
\hline Data size (voxel) & $(512,512,155)$ & $(512,512,165)$ & $(512,512,191)$ & $(512,512,221)$ \\
\hline Voxel size $(\mathrm{mm})$ & $0.683 \times 0.683 \times 1$ & $0.702 \times 0.702 \times 1$ & $0.613 \times 0.613 \times 1$ & $0.637 \times 0.637 \times 1$ \\
\hline $\begin{array}{l}\text { Running time without a lookup } \\
\text { table(s) }\end{array}$ & 158 & 383 & 209 & 278 \\
\hline $\begin{array}{l}\text { Running time with a lookup } \\
\text { table(s) }\end{array}$ & 74 & 160 & 97 & 119 \\
\hline Speedup factor & 2.14 & 2.39 & 2.15 & 2.34 \\
\hline Seed location & $(165,221,99)$ & $(197,190,108)$ & $(158,229,95)$ & $(189,233,137)$ \\
\hline Suggested threshold & 0.89 & 0.779 & 0.718 & 0.775 \\
\hline
\end{tabular}

Table 3 Comparisons between our method and the refined region-growing algorithm (RRG) presented in Ref. [1]

\begin{tabular}{lllll}
\hline Dataset & I & II & III & IV \\
\hline Data size (voxel) & $(512,512,155)$ & $(512,512,165)$ & $(512,512,191)$ & $(512,512,221)$ \\
$\begin{array}{l}\text { Seed location } \\
\text { Num of segmented vessel voxels using our } \\
\quad \text { method }\end{array}$ & $(165,221,99)$ & $(197,190,108)$ & $(158,229,95)$ & $(189,233,137)$ \\
$\quad$ Num of segmented vessel voxels using RRG & 28,918 & 45,624 & 111,399 & 89,631 \\
\hline
\end{tabular}


result of $A-B$ as shown in Figures 1e, 2e, 3e and 4e. According to the set $C$, our method based on fuzzy connectedness has advantage of detecting vascular edge. Due to the influence of partial volume effect, the edge of vessel is blurred and mixed with other tissues such as liver parenchyma, and $\mathrm{HU}$ values of vessels decrease from center to the edge. Therefore, it is hard for RRG to collect the edge voxels that should be belonged to the vessel. Besides, different vessel systems may have a weak connectivity in the vessel ends, thus it is able to generate more than one vessel system using only one seed as
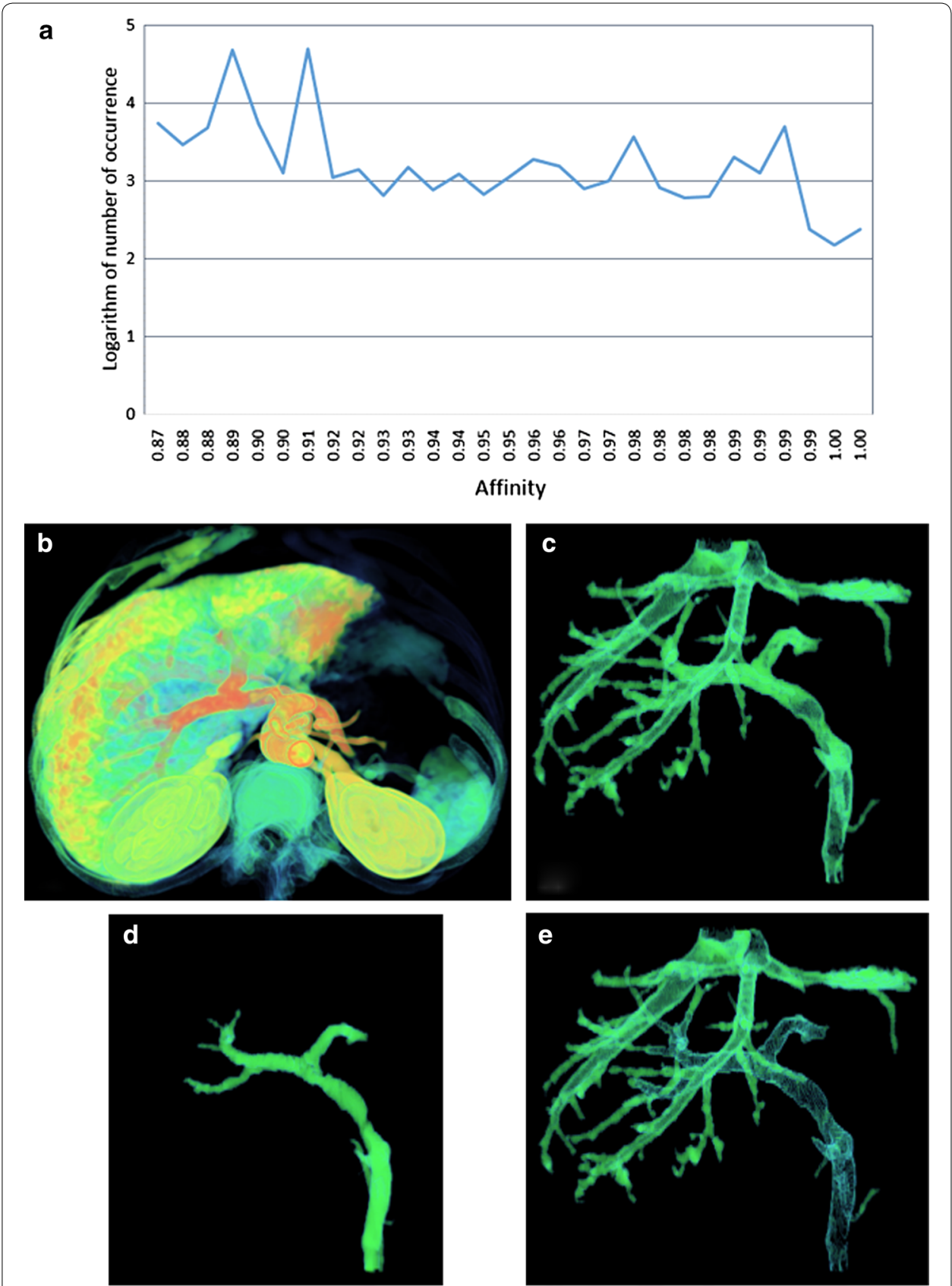

Figure 1 Segmentation results of dataset I: a histogram of the connectivity scene, $\mathbf{b}$ the fuzzy connectivity scene, $\mathbf{c}$ segmentation result using our method, $\mathbf{d}$ segmentation result using RRG, e the difference set $C$. 

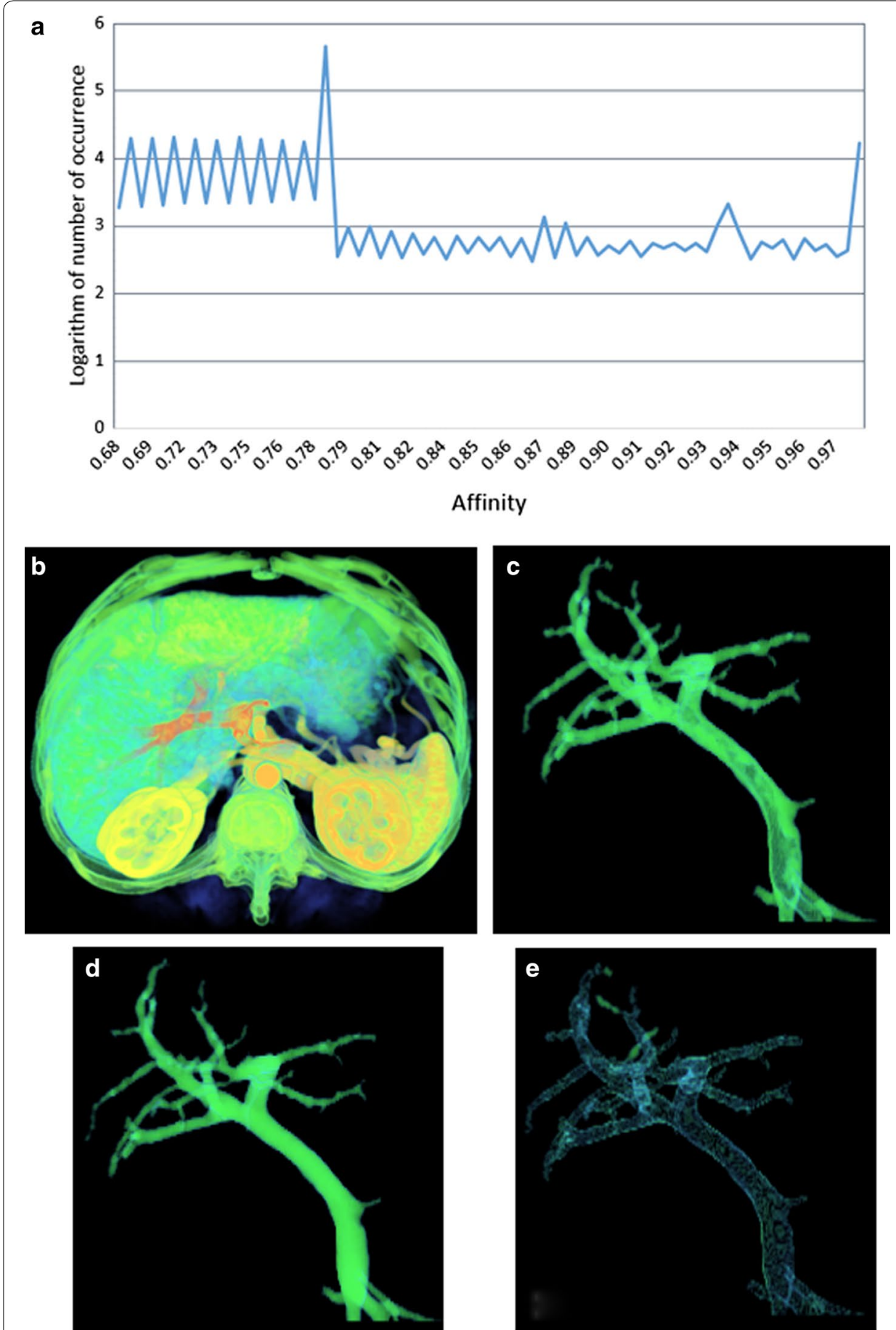

Figure $\mathbf{2}$ Segmentation results of dataset II: a histogram of the connectivity scene, $\mathbf{b}$ the fuzzy connectivity scene, $\mathbf{c}$ segmentation result using our method, $\mathbf{d}$ segmentation result using using RRG, e the difference set C.

shown in Figure 1c. According to Table 3 and set $C$, it is concluded that our method has better segmentation outcomes than RRG.

Figures 1, 2, 3 and 4 represent the histograms of the connectivity scenes, the corresponding connectivity scenes, the vessel segmentation results using fuzzy connected 


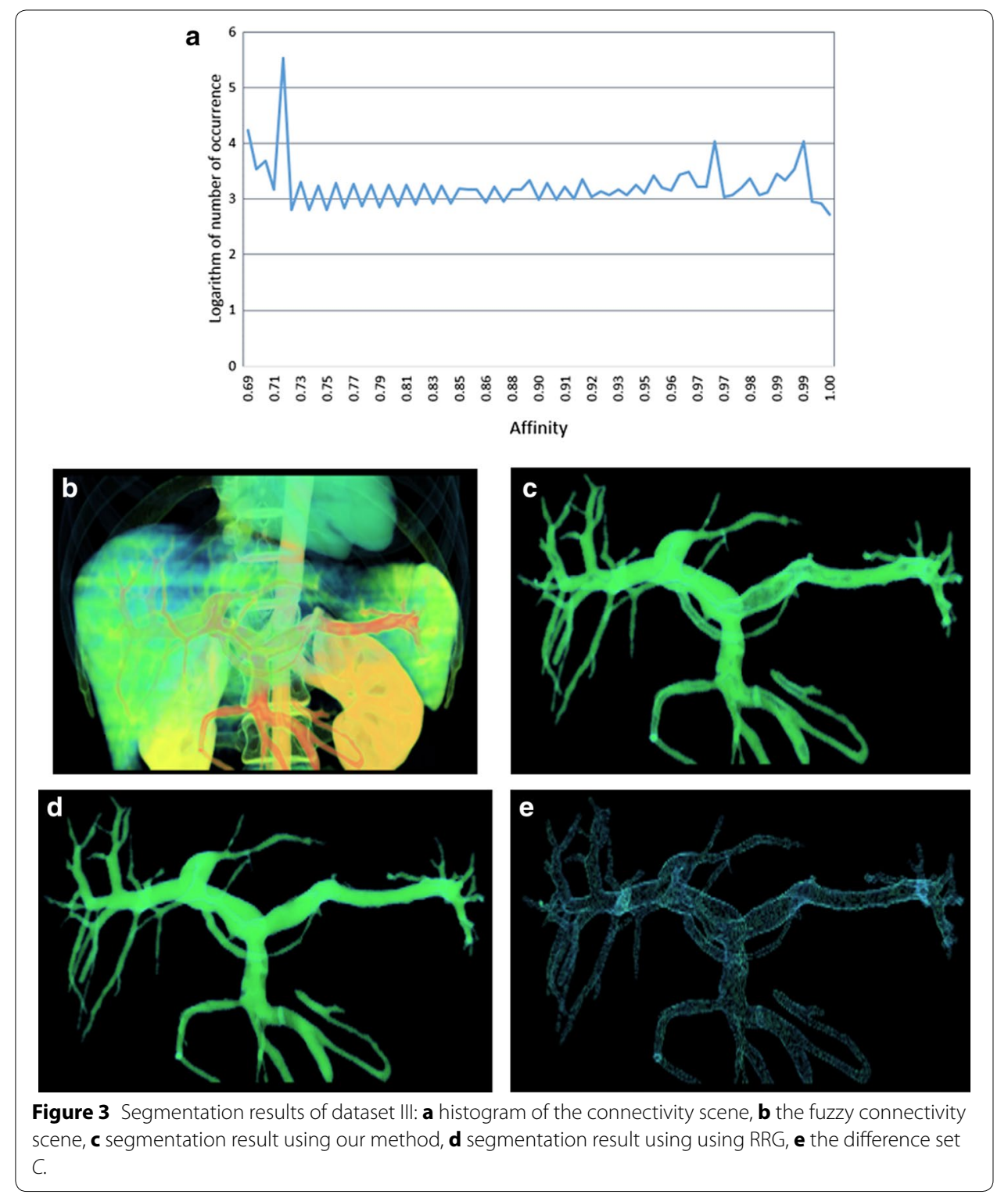

method and RRG, and the difference set $C$. In the histogram figures, the $\mathrm{X}$ axis represents affinity values which range from 0 to 1 , while the $Y$ axis represents the occurrence number (for convenience, in the form of its logarithm instead) of corresponding affinity value.

\section{Conclusions}

An improved algorithm based on fuzzy connectedness method was proposed in this paper. This algorithm uses an accelerated strategy based on a lookup table to reduce the cost of fuzzy connectedness calculation, and a watershed-like method for adaptive threshold searching automatically. Utilizing the replacement of the seed's value with mean value $m$, the algorithm is able to reduce the sensitivity to the location of the manually selected seed and to gain more robustness. Experiments based on four different datasets demonstrate 

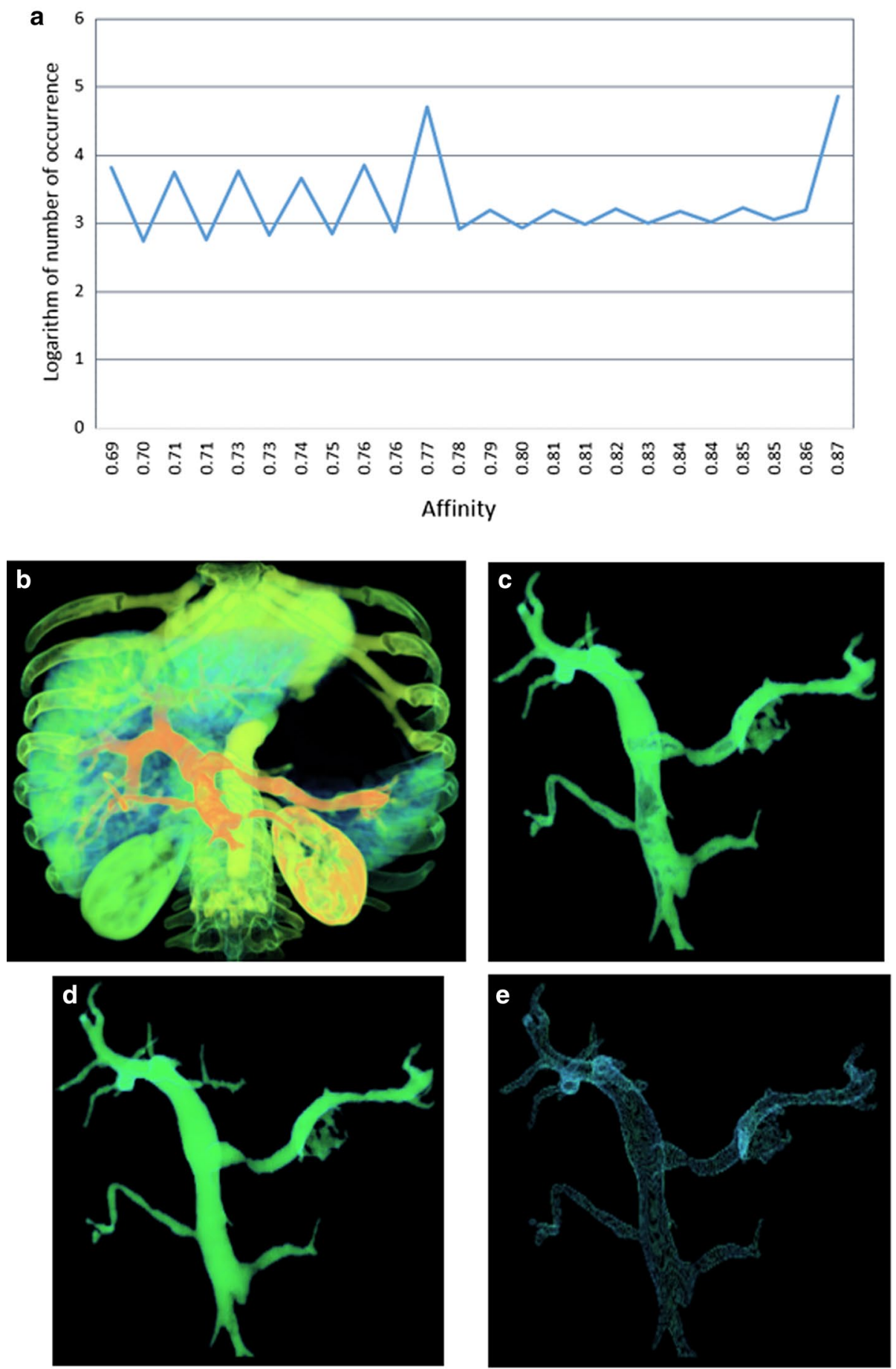

Figure 4 Segmentation results of dataset IV: a histogram of the connectivity scene, $\mathbf{b}$ the fuzzy connectivity scene, $\mathbf{c}$ segmentation result using our method, $\mathbf{d}$ segmentation result using using RRG, $\mathbf{e}$ the difference set C.

the efficiency of the lookup table method, which achieves a speed-up factor of above 2 . And the results also show that the adaptive threshold obtained automatically can always generate correct segmentation results. 
This algorithm has indicated an improvement in the performance of fuzzy connectedness method in hepatic vessel segmentation, but it is still not enough to achieve an interactive speed. A parallel version of the algorithm which uses Graphics Processing Unit (GPU) is currently being studied, and it is hopeful to achieve a faster speed, thus more efficiency.

Authors' contribution

XXG, SHH and XZF developed the algorithm, carried out the experiments and drafted the manuscript. BLW and XYH analyzed the data and provided suggestions and comments. All authors read and approved the final manuscript.

Author details

${ }^{1}$ Computer Science Department, Xiamen University, Xiamen, China. ${ }^{2}$ Computer Engineering College, Jimei University, Xiamen, China.

\section{Acknowledgements}

This work was supported by National Natural Science Foundation of China (Grant Nos. 61001144, 61102137 and 61327001) and the Natural Science Foundation of Fujian Province (Grant No. 2015J01264).

\section{Compliance with ethical guidelines}

\section{Competing interests}

The authors declare that they have no competing interests.

Received: 10 April 2015 Accepted: 9 June 2015

Published online: 19 June 2015

\section{References}

1. Selle D, Preim B, Schenk A, Peitgen HO. Analysis of vasculature for liver surgical planning. IEEE Trans Med Imaging. 2002;21(11):1344-57.

2. Foruzan AH, Zoroofi RA, Sato Y, Hori M. A Hessian-based filter for vascular segmentation of noisy hepatic CT scans. Int J Comput Assist Radiol Surg. 2012;7(2):199-205.

3. Esneault S, Lafon C, Dillenseger JL. Liver vessels segmentation using a hybrid geometrical moments/graph cuts method. IEEE Trans Biomed Eng. 2010;57(2):276-83.

4. Jiang $H Y$, He BC, Fang D, Ma ZY, Yang BQ, Zhang LB. A region growing vessel segmentation algorithm based on spectrum information. Comput Math Methods Med. 2013; doi:10.1155/2013/743870.

5. Wang Y, Fang B, Pi JR, Wu L, Wang PSP, Wang HG. Automatic multi-scale segmentation of intrahepatic vessel in CT images for liver surgery planning. Int J Patt Recogn Artif Intell. 2013;27(1):1-17.

6. Udupa JK, Samarasekera S. Fuzzy connectedness and object definition: theory, algorithm and applications in image segmentation. Graph Model Image Process. 1996;58(3):246-61.

7. Harati V, Khayati R, Farzan A. Fully automated tumor segmentation based on improved fuzzy connectedness algorithm in brain MR images. Comput Biol Med. 2011;41(7):483-92.

8. Lloréns R, Naranjo V, López F, Alcañiz M. Jaw tissues segmentation in dental 3D CT images using fuzzy-connectedness and morphological processing. Comput Methods Programs Biomed. 2012;108(2):832-43.

9. Ciesielski KC, Miranda PA, Falcão AX, Udupa JK. Joint graph cut and relative fuzzy connectedness image segmentation algorithm. Med Image Anal. 2013;17(8):1046-57.

10. Badura P, Pietka E. Soft computing approach to 3D lung nodule segmentation in CT. Comput Biol Med. 2014:53:230-43.

11. Nyúl LG, Falcão AX, Udupa JK. Fuzzy-connected 3D image segmentation at interactive speeds. Graph Models. 2002;64(5):259-81.

12. Ostu N. A threshold selection method from gray-level histograms. IEEE Trans Syst Man Cybern. 1979;9(1):62-6. 